\section{Propuesta de un glosario para la discusión del aborto}

\author{
CARMEN ASTETE A., JUAN PABLO BECA I., ALBERTO LECAROS U. ${ }^{a}$
}

\section{A glossary for discussion about abortion}

Abortion and its diverse possible legal regulations is one of the major and toughest social controversies. This debate is even more problematic due to biases, prejudgments, different ideologies, beliefs, religious doctrines and political pressures. Chile has recently begun a new national discussion with an evident confusion, both in juridical and clinical terminology, which makes very difficult to achieve the necessary plural debate for a social and political consensus. The authors structured an academic collaborative project to create a glossary as a contribution for a discussion based on clearly defined notions about the different terms used in the abortion debate. Twenty-two concepts were selected and their definitions were reviewed and discussed by more than 50 different specialists. The final version of this glossary in Spanish language is presented.

(Rev Med Chile 2014; 142: 1449-1451)

Key words: Abortion, eugenic; Abortion, induced; Abortion, legal.

\author{
Centro de Bioética. Facultad \\ de Medicina Clínica Alemana \\ Universidad del Desarrollo. \\ Santiago-Chile. \\ ${ }^{a}$ Abogado. PhD@ en Filosofía. \\ Universidad Complutense de \\ Madrid. \\ Recibido el 3 de septiembre de \\ 2014, aceptado el 29 de octubre \\ de 2014. \\ Correspondencia a: \\ Dra. Carmen Astete \\ Centro de Bioética. \\ Facultad de Medicina Clínica \\ Alemana. Universidad del \\ Desarrollo. Santiago-Chile. \\ castete@udd.cl
}

L a legalización o despenalización del aborto constituye un tema que en todos los países genera controversias de mucha complejidad. La diferencia entre legalización y despenalización admite diferentes interpretaciones, aun cuando lo relevante es que la despenalización se refiere sólo a eliminar el carácter punible del acto.

El debate sobre el aborto se exacerba frecuentemente por sesgos, juicios previos, ideologías, presiones políticas, doctrinarias y religiosas. Es así como se han originado campañas polarizadas de grupos que defienden o rechazan las diferentes propuestas regulatorias o legislativas, incluyendo movimientos como los llamados "pro-choice" versus los llamados "pro-life". En nuestro medio la discusión social sobre el aborto se ha dado con características similares y la sociedad ha opinado de manera muy variada según lo demuestran encuestas recientes ${ }^{1,2}$. En el último tiempo, a raíz de anuncios gubernamentales de una próxima discusión a nivel parlamentario del aborto en Chile, actualmente penalizado en cualquier circunstancia, se ha generado un fuerte debate público que ha dejado en evidencia, no sólo una esperable carga doctrinaria y política, sino también una confusión conceptual en el uso de la terminología. La falta de precisión conceptual dificulta aún más la posibilidad de un debate plural y constructivo. Este necesario debate público se prolongará probablemente por los próximos meses o años. Con el propósito de contribuir a que la discusión sobre el aborto se realice con la necesaria claridad y precisión conceptual, lo cual favorece el debido respeto a la diversidad de opiniones, y al logro de consensos, hemos realizado un trabajo académico colaborativo para proponer un glosario que defina la terminología médica y jurídica pertinente a la discusión a nivel nacional. Estas definiciones incluyen tanto aspectos biológicos como clínicos y éticos.

Para seleccionar y luego elaborar la definición de los términos que se refieren al aborto, se confeccionó un primer listado en base a una revisión de publicaciones nacionales e internacionales sobre el tema ${ }^{3-9}$. Este primer documento fue revisado por un grupo de profesionales relacionados al tema, después de lo cual se envió a los jefes de servicio de Obstetricia y Ginecología del país y fue subido a la página web del Centro de Bioética de la Facultad de Medicina Clínica Alemana Universidad del 
Desarrollo para recibir comentarios y sugerencias. Después de un plazo de 45 días de esta consulta pública, habiendo acogido las sugerencias recibidas, el glosario fue analizado en una reunión abierta de 50 profesionales interesados donde se discutieron las definiciones sugeridas. La conclusión de este proceso participativo de estudio se presenta como un glosario para la discusión del aborto, destinado a facilitar una discusión social, plural y respetuosa de este relevante tema de interés público.

\section{Glosario}

\section{Aborto}

La Organización Mundial de la Salud define el aborto como la interrupción del embarazo cuando el embrión o feto todavía no es viable fuera del vientre materno (ver Viabilidad fetal).

Después de la viabilidad, la interrupción del embarazo se considera inducción de parto prematuro. Si la intención es la eliminación del feto se considera aborto tardío (late-term abortion).

Aborto en sentido jurídico es toda maniobra destinada a interrumpir el embarazo impidiendo que llegue al término natural, con destrucción o muerte del producto.

\section{Aborto despenalizado}

Es el aborto que se realiza bajo un régimen legal que ha eliminado su carácter punible en circunstancias determinadas.

\section{Aborto espontáneo}

Es el aborto que ocurre de manera natural, sin mediar la intención de hacerlo.

\section{Aborto eugenésico}

Es el aborto inducido con la intención de evitar el nacimiento de un niño portador de una anomalía fetal incompatible con la vida post-natal, o de de un niño severamente malformado. Estos casos se definen equívocamente como "aborto por feto inviable".

\section{Aborto ilegal}

Es el aborto realizado en contra de las leyes del país donde se practica.

\section{Aborto indirecto}

Se refiere al aborto que ocurre como consecuencia de una intervención cuyo fin es tratar una patología materna aunque la pérdida fetal, sin ser buscada, se hubiere previsto.

\section{Aborto inseguro o peligroso}

La OMS lo define como el aborto llevado a cabo por personas que carecen de las habilidades necesarias, o en un ambiente que no cumple con mínimos estándares médicos.

\section{Aborto legal}

Es el aborto inducido cuando se realiza en un país cuyo régimen legal lo permite.

\section{Aborto libre o a demanda}

Es el aborto que se realiza bajo un régimen legal que lo permite por la sola petición de la mujer, sin que concurran circunstancias o condiciones especiales que lo autoricen.

\section{Aborto por incesto}

Es el aborto inducido cuando el embarazo se ha originado como consecuencia de relaciones sexuales abusivas entre parientes.

\section{Aborto por violación}

Es el aborto inducido cuando el embarazo se ha originado como consecuencia de un hecho constitutivo del delito de violación, independientemente de la edad de la mujer.

\section{Aborto provocado o inducido}

Es la interrupción deliberada del embarazo con feto no viable como resultado de intervenciones físicas, instrumentales o medicamentosas. Las intervenciones pueden ser realizadas por la propia embarazada o por otra persona.

\section{Aborto terapéutico}

Es el aborto inducido con la intención de proteger la salud o la vida de la embarazada cuando éstas se encuentran en grave riesgo.

\section{Embarazo}

Es la condición de la mujer que se extiende entre la implantación del blastocisto en el endometrio y el parto. Sin embargo la edad gestacional del feto se determina a contar del primer día de la última regla.

\section{Embarazo ectópico}

Es el embarazo en el cual el embrión se implanta fuera de la cavidad uterina, usualmente en 
Propuesta de un glosario para la discusión del aborto - C. Astete et al

la trompa de Falopio. Es una condición de riesgo de vida para la madre si no se detecta y se trata oportunamente. Sinónimos: embarazo tubario y embarazo extra-uterino.

\section{Embrión humano}

Es el producto de la concepción en su fase inicial de desarrollo, desde la etapa de zigoto hasta las 8 semanas después de la fecundación.

\section{Feto humano}

Es el producto de la concepción en desarrollo, desde las 8 semanas después de la fecundación hasta el momento del parto en que se convierte en un recién nacido.

\section{Interrupción del embarazo}

Es la intervención instrumental y/o medicamentosa para poner fin a un embarazo. La consecuencia de dicha intervención es el aborto si es antes de la viabilidad fetal, o la inducción de un parto prematuro si se realiza después.

\section{Nasciturus}

Término jurídico que designa al ser humano desde que es concebido hasta su nacimiento, esto es antes de su separación completa de la madre.

\section{Objeción de conciencia}

Consiste en la oposición de una persona a un imperativo legal o de una autoridad, basada en sus propios principios morales. Se aplica por lo tanto de manera personal para actos específicos, con el debido fundamento previamente expresado.

Las instituciones no tienen derecho a objeción de conciencia, aunque pueden adherir a valores que se expresan en sus estatutos y normativas.

\section{Viabilidad fetal}

Es la edad gestacional a partir de la cual es posible la vida extrauterina. La OMS define este límite en las 20 semanas de edad gestacional (18 semanas después de la fecundación), o en más de 400 grs. si la edad gestacional es desconocida. Sin embargo, el límite de viabilidad depende del progreso médico y tecnológico, estando actualmente en torno a las 23 semanas de gestación o en 500 gr. de peso al nacer.

\section{Referencias}

1. Encuesta UDP, 2010. Disponible en http://www. encuesta.udp.cl/wp-content/uploads/2010/12/ICSOAborto-2010.pdf

2. Dides C, Benavente MC, Saez I. Estado del arte sobre aborto en Brasil, Chile, Mexico y Nicaragua. Editorial Flacso-Chile 2011. Disponible en http://issuu.com/ flacso.chile/docs/estado_del_arte_parte1

3. Zegers-Hochschild F, Adamson GD, de Mouzon J, Ishihara $\mathrm{O}$, Mansour R, Nygren $\mathrm{K}$ et al. on behalf of ICMART and WHO. Glosario de terminología en Técnicas de Reproducción Asistida (TRA). Versión revisada y preparada por el International Committee for Monitoring Assisted Reproductive Technology (ICMART) y la Organización Mundial de la Salud (OMS). OMS 2009. Disponible en: http://www.who.int/reproductivehealth/ publications/infertility/art_terminology2/es/ [Consultado el 23 de Julio 2014].

4. Ley Orgánica 2/2010de salud sexual y reproductiva y de la interrupción voluntaria del embarazo. España.

5. Comité Etica Soc Chil Pediatría. El aborto terapéutico. Rev Chil Pediatr 1999; 70: 86-87.

6. Aspectos médicos y jurídicos del aborto. Bioetica\&Debat 2008; 51: 1-18.

7. Glosario de IPPF sobre términos relacionados con la salud sexual y reproductiva. International Planned Parenthood Federation. 2010. Disponible en http://www. ippf.org/

8. Beca JP. La discusión del aborto en Chile. Rev Chil Pediatr 2014; 85: 418-20.

9. Jensen D. Birth, meaningful viability and abortion. J Med Ethics 2014; on-line July 10: 10.1136/medethics-2013-101911. 